Tropical Journal of Pharmaceutical Research April 2015; 14 (4): 583-590

ISSN: $1596-5996$ (print); 1596-9827 (electronic)

(C) Pharmacotherapy Group, Faculty of Pharmacy, University of Benin, Benin City, 300001 Nigeria.

All rights reserved.

Available online at http://www.tjpr.org

Original Research Article

http://dx.doi.org/10.4314/tjpr.v14i4.4

\title{
Formulation and Development of Dendrimer-Based Transdermal Patches of Meloxicam for the Management of Arthritis
}

\author{
Xiang-Dong Duan ${ }^{1,2}$, Chang-Jiao $\mathrm{Ji}^{1,2}$ and Lin $\mathrm{Nie}^{1 *}$ \\ ${ }^{1}$ Department of Orthopaedics, Qilu Hospital of Shandong University, ${ }^{2}$ Department of Orthopaedics, Affiliated Hospital of \\ Shandong Traditional Chinese Medicine University, Jinan 250012, China
}

*For correspondence: Email: nielin687@gmail.com; Tel/Fax: 0086-531-68617065

Received: 1 August 2014

Revised accepted: 28 February 2015

\begin{abstract}
Purpose: To develop transdermal patches of meloxicam (MLX) using chitosan and hydroxypropyl methylcellulose (HPMC) and polyvinyl alcohol (PVA) as hydrophilic polymers, polyamido amine (PAMAM) dendrimer as a permeation enhancer, and dibutyl pthalate as a plasticizer

Methods: The patches were prepared by solvent casting evaporation technique using 3-factor, 3-level Box-Behnken design. The patches were evaluated for physical appearance, thickness, weight variation, folding endurance, drug content uniformity, tensile strength, moisture absorption and moisture loss, in vitro drug release, as well as by field-emission scanning electron microscopy (FESEM) and $x$-ray diffraction (XRD). A specially designed glass diffusion cell was used for the in vitro drug release study. The effect of concentrations of dependent variables (PAMAM G3, chitosan and dibutyl pthalate) on drug release was investigated.

Results: The patches demonstrated satisfactory characteristics. PAMAM dendrimer significantly enhanced $(p<0.5)$ the permeation of MLX. A maximum of $85.7 \%$ drug release was achieved in $24 \mathrm{~h}$.

Conclusion: Dendrimer increased the release of MLX by increasing its solubility and permeation through the membrane. Thus, dendrimer patches are a potentially suitable transdermal drug delivery system for the management of some diseased conditions.
\end{abstract}

Keywords: Dendrimers, Transdermal patches, Skin permeation, Permeation enhancer, Chitosan, Hydroxypropyl methyl cellulose, Meloxicam, Plasticizer

Tropical Journal of Pharmaceutical Research is indexed by Science Citation Index (SciSearch), Scopus, International Pharmaceutical Abstract, Chemical Abstracts, Embase, Index Copernicus, EBSCO, African Index Medicus, JournalSeek, Journal Citation Reports/Science Edition, Directory of Open Access Journals (DOAJ), African Journal Online, Bioline International, Open-J-Gate and Pharmacy Abstracts

\section{INTRODUCTION}

Arthritis is an autoimmune disease and disorder of the joints characterized by inflammation of one or more joints of the body part $[1,2]$. The use of NSAID's is ubiquitous in the management of arthritic condition due to their effectiveness as anti-inflammatory and analgesic activity [3]. Meloxicam (MLX) is a oxicam derivative, is a member of the enolic acid group of NSAID's. MLX has been effectively used in symptomatic management of the rheumatoid arthritis and osteo arthritis. MLX have lower toxicity than the other NSAIDs with similar efficacy for reducing the pain and anti-inflammatory symptoms. NSAIDs including MLX is characterized by their gastrointestinal adverse effects which include ulceration, bleeding, inflammation and perforation of the stomach. So oral route is not obvious route for the administration of MLX. Considering all these issues it's become mandatory to develop other drug delivery system 
other than oral to overcome the problems associated with current delivery technology. Dendrimers are potential permeation enhancer as well as solubility enhancer $[4,5]$.

Dendrimers are monodisperse, hyper branched, three dimensional, tree-like macromolecules having host-guest entrapment properties. Wang et al studied novel transdermal drug delivery system with polyhydroxyalkanoante (PHA) and starburst polyamidoamine dendrimers [6]. Chauhan et al studied dendrimers mediated transdermal delivery to enhance bioavailability of indomethacin [7]. Vamsi et al investigated the effect of poly(amidoamine) (PAMAM) dendrimer on skin permeation of 5-fluorouracil [8].

Transdermal drug delivery system (TDDS) in the form of patch is an innovative technique for the application on skin to achieve systemic effects. The aim of this study was to develop an effective transdermal patch using dendrimer that would avoid the adverse effects of oral MLX.

\section{EXPERIMENTAL}

\section{Materials}

Meloxicam (MLX) was purchased from Shouguang Fukang Pharmacy Factory (Shandong, China), Polyamido amine dendrimer Generation 3 (PAMAM G3) was purchased from Sigma Aldrich, USA. Chitosan, hydroxypropyl methylcellulose (HPMC), polyvinyl alcohol (PVA), dibutyl pthalate, acetic acid and methanol were purchased from Shanghai Chemical C. (Shanghai, China).

\section{Preparation of transdermal patch}

Solvent casting evaporation was performed to prepare the patches on glass petri dish as described previously [9]. PVA (2 \% dispersion) was prepared by dispersing the polymer in double-distilled water $(20 \mathrm{ml})$ and heating to 40 $0 \mathrm{C}$. The baking membrane was cast by pouring PVA dispersion $(20 \mathrm{ml})$ on to the petri dish followed by drying in hot air oven at $55^{\circ} \mathrm{C}$ for $8 \mathrm{~h}$. The drug reservoir compartment was prepared by dissolving HPMC (1.5\%) in double distilled water $(25 \mathrm{ml})$. To this solution, dibutyl Pthalate (10, 20 and $30 \%)$ (plasticizer) and various concentrations of PAMAM dendrimer $(0.3,0.45$ and $0.6 \%$ ) was added. MLX (100 mg) dissolved in methanol $(10 \mathrm{ml})$ was added drop by drop to the HPMC-plasticizer dispersion under slow stirring with magnetic stirrer. The whole dispersion containing the drug was cast on the PVA backing membrane and allowed to dry for 6 $\mathrm{h}$ at $50{ }^{\circ} \mathrm{C}$. The rate controlling membrane (consisting of chitosan) was prepared by dissolving the polymer in $2 \%$ acetic acid, cast on the drug reservoir, followed by drying at $50{ }^{\circ} \mathrm{C}$ for $4 \mathrm{~h}$. The dry patch was removed from Petri dish and cut into small patches of $1 \mathrm{~cm}^{2}$ and kept in a desiccator containing silica as desiccant and used for further analysis. The composition of the formulations are shown in Table 1.

\section{Experimental design}

Experiments were designed using Design Expert 6.0.8 portable Stat-Ease, Inc. software. A 3factor, 3-level Box-Behnken design was used to derive a second order polynomial equation and construct contour plots to predict responses

\section{Evaluation of transdermal patches}

All patches were examined visually and inspected for smoothness, color and clarity. Thickness was measured at 5 different locations by using screw gauze and average thickness was determined. Individually, 10 patches from each batch were weighed and the mean weight was determined. Folding endurance test was performed to determine the strength of the patch and to check the efficiency of the plasticizer. It was determined by repeatedly folding the patch at the same place until it broke. The number of times the patch could be folded at the same place without breaking or cracking gave the value of folding endurance [10].

Drug content uniformity test was performed on three patches. Each patch was soaked and dissolved in $50 \mathrm{ml}$ of methanol and resulting solution was filtered to remove undissolved residue. Aliquots were prepared and measured spectrophotometrically for drug content at 365 $\mathrm{nm}$. [9]. Mechanical properties and strength of the patches were determined by measuring their tensile strength. Tensile strength of the patch was determined with Universal strength testing apparatus (Hounsfield, Slinfold, Horsham, U.K.) [11].

Moisture uptake was determined by first weighing each patch accurately and then placed in a desiccator containing silica as a desiccant. After 5 days, the patches were taken out and weighed individually. Percent moisture uptake was calculated as the difference between final and initial weight with respect to initial weight [12]. For moisture content determination, $t$, the patches were weighed accurately and kept in a desiccator containing anhydrous calcium chloride. After 5 days, the patches were taken out and weighed. 
Table 1: Variable and three levels, and formulation design

\begin{tabular}{|c|c|c|c|c|c|c|}
\hline \multicolumn{3}{|c|}{ Independent variable } & Low level (-1) & \multicolumn{2}{|c|}{ Medium level (0) } & High level (+1) \\
\hline \multicolumn{2}{|c|}{$A=P A M A M$ conc. $(\%)$} & & 0.3 & \multicolumn{2}{|c|}{0.45} & 0.6 \\
\hline \multicolumn{2}{|c|}{$B=$ Chitosan conc. $(\%)$} & & 1 & \multicolumn{2}{|r|}{2} & 3 \\
\hline & & 10 & \multicolumn{2}{|r|}{20} & 30 \\
\hline \multicolumn{7}{|c|}{$\begin{array}{l}\text { Dependent Variables } \\
\text { Y1 = \% DR (drug release) }\end{array}$} \\
\hline \multicolumn{7}{|c|}{ Composition of and Diagnostic Statistics for Y1 } \\
\hline \multirow[t]{2}{*}{ Batch } & & Factor & & Actual DR & Predicted DR & Residual \\
\hline & A & B & C & Y1 & & \\
\hline F1 & -1 & 0 & +1 & 33.25 & 34.79 & -1.54 \\
\hline $\mathbf{F 2}$ & 0 & 0 & 0 & 52.57 & 56.21 & -3.64 \\
\hline F3 & 0 & 0 & 0 & 50.24 & 56.21 & -5.97 \\
\hline F4 & 0 & -1 & -1 & 55.78 & 60.68 & -4.90 \\
\hline F5 & +1 & 0 & +1 & 69.9 & 73.67 & -3.77 \\
\hline F6 & -1 & +1 & 0 & 30.4 & 34.28 & -3.88 \\
\hline F7 & 0 & 2 & 0 & 51.78 & 56.21 & -4.43 \\
\hline F8 & +1 & +1 & 0 & 72.65 & 73.16 & -0.51 \\
\hline F9 & 0 & -1 & +1 & 59.67 & 56.72 & 2.95 \\
\hline F10 & 0 & +1 & -1 & 62.86 & 55.70 & 7.16 \\
\hline F11 & -1 & 0 & -1 & 42.23 & 38.75 & 3.48 \\
\hline F12 & 0 & +1 & +1 & 60.45 & 51.74 & 8.71 \\
\hline F13 & 0 & 0 & 0 & 52.38 & 56.21 & -3.83 \\
\hline F14 & +1 & 0 & -1 & 78.24 & 77.63 & 0.61 \\
\hline F15 & +1 & 1 & 0 & 85.71 & 78.14 & 7.57 \\
\hline F16 & 0 & 0 & 0 & 52.35 & 56.21 & -3.86 \\
\hline F17 & -1 & -1 & 0 & 45.1 & 39.26 & 5.84 \\
\hline
\end{tabular}

Percent moisture loss (which is a measure of moisture content) is generally used to determine whether a patch has sufficient. Patches with very less moisture content tend to become brittle in nature was calculated as the difference between final and initial weight with respect to initial weight [12].

Specially designed glass diffusion cell was used to carry out in vitro release study. Cellophane dialysis membrane was soaked previously for 24 $h$ in $\mathrm{pH} 7.5$ phosphate buffer [13]. This membrane was stretched around one end of the diffusion cell. The working surface area of the membrane was $2.5 \mathrm{~cm}^{2}$. The tube (donor compartment) was immersed in a glass beaker containing $\mathrm{pH} 7.5$ phosphate buffer (receptor compartment) in such a way that the membrane just touched the receptor medium. A weighed transdermal patch $\left(1 \mathrm{~cm}^{2}\right)$ was placed on the cellophane dialysis membrane. The receptor medium was maintained at $37 \pm 0.5{ }^{\circ} \mathrm{C}$ and stirred at $50 \mathrm{rpm}$ by keeping it on hot plate magnetic stirrer. Aliquots $(5 \mathrm{ml})$ of samples were withdrawn from the receptor medium at predetermined time intervals and replaced with equal volume of fresh buffer to maintain sink conditions. Samples were analysed with spectrophotometrically at $365 \mathrm{~nm}$ and drug release was computed.
Surface morphology of the drug- loaded patches were studied using scanning electron microscopy (FESEM-S 4800, Hitachi, Japan).

The crystalline and amorphous nature of MLX, chitosan, HPMC and drug-loaded patch were analysed by -ray diffraction (Miniflex, Rikagu).

\section{RESULTS}

\section{Physical characteristics of patches}

F2, F4, F5, F9 were smooth on their surface while F10, F3, F6, F8, F12, F14, F16 were rough. All data is shown in Table 2. Some of the batches were found to be transparent in nature. The thickness of the patches was found to be in the range of $0.02 \pm 0.15$ to $0.04 \pm 0.28 \mathrm{~mm}$. The tensile strength of the patches was found in the range of $0.30 \pm 0.0040$ to $0.62 \pm 0.38 \mathrm{~kg} / \mathrm{cm}^{2}$. The weight of patches was found to be in the range of $0.13 \pm 0.25 \mathrm{gm}$ to $0.16 \pm 0.30 \mathrm{gm}$. Also the uniformity of drug content of drug among all the batches patches were observed and ranged from $95.36 \pm 1.15$ to $98.47 \pm 0.91 \%$ shown in. Folding endurance of the hydrophilic chitosan/HPMC based patches ranged from $132.24 \pm 3.21$ to $189.14 \pm 3.24$. Moisture absorption was in the range of $1.23 \pm 0.25$ to $3.72 \pm 0.28 \%$. 
Table 2: Physical characteristics of patches

\begin{tabular}{|c|c|c|c|c|c|}
\hline Batch & Appearance & $\begin{array}{c}\text { Folding } \\
\text { endurance }\end{array}$ & $\begin{array}{l}\text { Tensile strength } \\
\left(\mathrm{kg} / \mathrm{cm}^{2}\right)\end{array}$ & $\begin{array}{c}\text { Moisture } \\
\text { absorption (\%) }\end{array}$ & $\begin{array}{c}\text { Moisture loss } \\
(\%)\end{array}$ \\
\hline F1 & $\bar{T}$ & $138.20 \pm 2.25$ & $0.30 \pm 0.01$ & $1.70 \pm 0.22$ & $1.20 \pm 0.25$ \\
\hline F2 & $S$ & $150.21 \pm 3.20$ & $0.31 \pm 0.38$ & $1.69 \pm 0.20$ & $2.30 \pm 0.27$ \\
\hline F3 & $\mathrm{R}$ & $165.25 \pm 3.26$ & $0.39 \pm 0.37$ & $2.56 \pm 0.21$ & $1.24 \pm 0.30$ \\
\hline F4 & $S$ & $145.20 \pm 4.30$ & $0.40 \pm 0.30$ & $2.55 \pm 0.25$ & $1.32 \pm 0.35$ \\
\hline F5 & S & $178.23 \pm 3.15$ & $0.60 \pm 0.42$ & $1.70 \pm 0.27$ & $1.28 \pm 0.32$ \\
\hline F6 & $\mathrm{R}$ & $168.43 \pm 3.25$ & $0.62 \pm 0.38$ & $3.69 \pm 0.12$ & $3.23 \pm 0.42$ \\
\hline F7 & $\mathrm{T}$ & $152.54 \pm 3.28$ & $0.56 \pm 0.20$ & $2.45 \pm 0.15$ & $3.31 \pm 0.57$ \\
\hline F8 & $\mathrm{R}$ & $189.14 \pm 3.24$ & $0.58 \pm 0.54$ & $3.50 \pm 0.22$ & $3.50 \pm 0.45$ \\
\hline F9 & $S$ & $165.25 \pm 3.23$ & $0.55 \pm 0.89$ & $2.56 \pm 0.23$ & $2.21 \pm 0.29$ \\
\hline F10 & $S$ & $139.25 \pm 3.37$ & $0.35 \pm 0.40$ & $3.22 \pm 0.22$ & $2.20 \pm 0.15$ \\
\hline F11 & $\mathrm{T}$ & $125.33 \pm 3.51$ & $0.36 \pm 0.45$ & $3.70 \pm 0.26$ & $3.24 \pm 0.25$ \\
\hline F12 & $\mathrm{R}$ & $132.24 \pm 3.21$ & $0.38 \pm 0.59$ & $1.56 \pm 0.11$ & $1.19 \pm 0.53$ \\
\hline F13 & $\mathrm{T}$ & $148.20 \pm 3.27$ & $0.59 \pm 0.23$ & $2.40 \pm 0.27$ & $1.20 \pm 0.55$ \\
\hline F14 & $\mathrm{R}$ & $175.23 \pm 3.32$ & $0.40 \pm 0.25$ & $3.72 \pm 0.28$ & $1.28 \pm 0.51$ \\
\hline F15 & $\mathrm{T}$ & $169.35 \pm 3.39$ & $0.34 \pm 0.24$ & $1.23 \pm 0.25$ & $4.12 \pm 0.24$ \\
\hline F16 & R & $170.36 \pm 3.24$ & $0.33 \pm 0.28$ & $1.72 \pm 0.30$ & $2.50 \pm 0.21$ \\
\hline F17 & $\mathrm{T}$ & $185.30 \pm 3.26$ & $0.45 \pm 0.45$ & $1.65 \pm 0.28$ & $2.70 \pm 0.25$ \\
\hline
\end{tabular}

Note: $T=$ transparent, $S=$ smooth, $R=$ rough

\section{Optimized in vitro drug release}

In vitro drug release was performed in $\mathrm{pH} 7.4$ phosphate buffer using a modified diffusion cell. Drug release in $24 \mathrm{~h}$ was in the range of 33.25 (F1) to $85.71 \%$ (F15). The polynomial equation obtained for DR (Y1) is given by Eq 1 .

$Y 1=+56.21+19.44 \mathrm{~A}-2.49 \mathrm{~B}-1.98 \mathrm{C}$

A positive value in Eq 1 equation represents the synergistic effect of the independent variable. + 19.44A represents the positive or synergistic effect of independent factor A (dendrimer concentration) on in vitro drug release. A negative value represents the inverse relationship (antagonistic effect). The value, $2.49 \mathrm{~B}$, represents the antagonistic effect of independent factor $B$ (chitosan concentration) on in vitro drug release. The suggested model for the $\mathrm{Y} 1$ was linear as shown in Table 3. The value of correlation coefficient $\left(R^{2}\right)$ for the response $Y 1$ was 0.8874 which indicates the good fit for the linear model. The $p$-value for the Y1 model was found to be $<0.0001$ which clearly indicates the model is statistically significant. Also among the three independent variables selected only factor A (Dendrimer Conc.) was found to be statistically significant $(p<0.0001)$ while the effect of factor B $(p=0.2234)$ and C $(p=0.3274)$ was not statistically significant (Table 3 ). Diagnostics case statistics for various response variables are shown in Table 3 . The drug release pattern shown by the patches had wide variation from $33.25 \%$ (F1) to $85.71 \%$ (F15). A comparative in vitro drug release is shown in Figure 1.

Table 3: Regression analysis for response $Y 1$ and ANOVA of models

\begin{tabular}{|c|c|c|c|c|c|c|}
\hline Models & $\mathbf{R}^{2}$ & Adjusted $\mathrm{R}^{2}$ & Predicted $\mathrm{R}^{2}$ & Std. Dev & Press & Remarks \\
\hline \multicolumn{7}{|l|}{ Response Y1 } \\
\hline Linear & $\underline{0.8874}$ & $\underline{0.8615}$ & $\underline{0.7914}$ & $\underline{5.50}$ & $\underline{729.59}$ & Suggested \\
\hline $2 \mathrm{FI}$ & $\overline{0.8905}$ & $\overline{0.8248}$ & $\overline{0.5498}$ & $\overline{6.19}$ & $\overline{1574.77}$ & .... \\
\hline Quadratic & 0.9373 & 0.8566 & 0.0113 & 5.60 & 3458.22 & \\
\hline Cubic & 0.9990 & 0.9958 & & 0.95 & + & Aliased \\
\hline \multicolumn{7}{|c|}{ Regression equations of the fitted model: $Y 1=+56.21+19.44 A-2.49 B-1.98 C$} \\
\hline \multicolumn{7}{|c|}{ ANNOVA for response $\mathrm{Y1}$} \\
\hline Source & DF & Sum of squares & Me & square & F value & $P$-value \\
\hline Model for Y1 & 3 & 3104.17 & & 4.72 & 34.17 & $<0.0001$ \\
\hline A & 1 & 3023.31 & & 3.31 & 99.83 & $\leq 0.0001$ \\
\hline B & 1 & 49.50 & & 50 & 1.63 & 0.2234 \\
\hline C & 1 & 31.36 & & 36 & 1.04 & 0.3274 \\
\hline
\end{tabular}




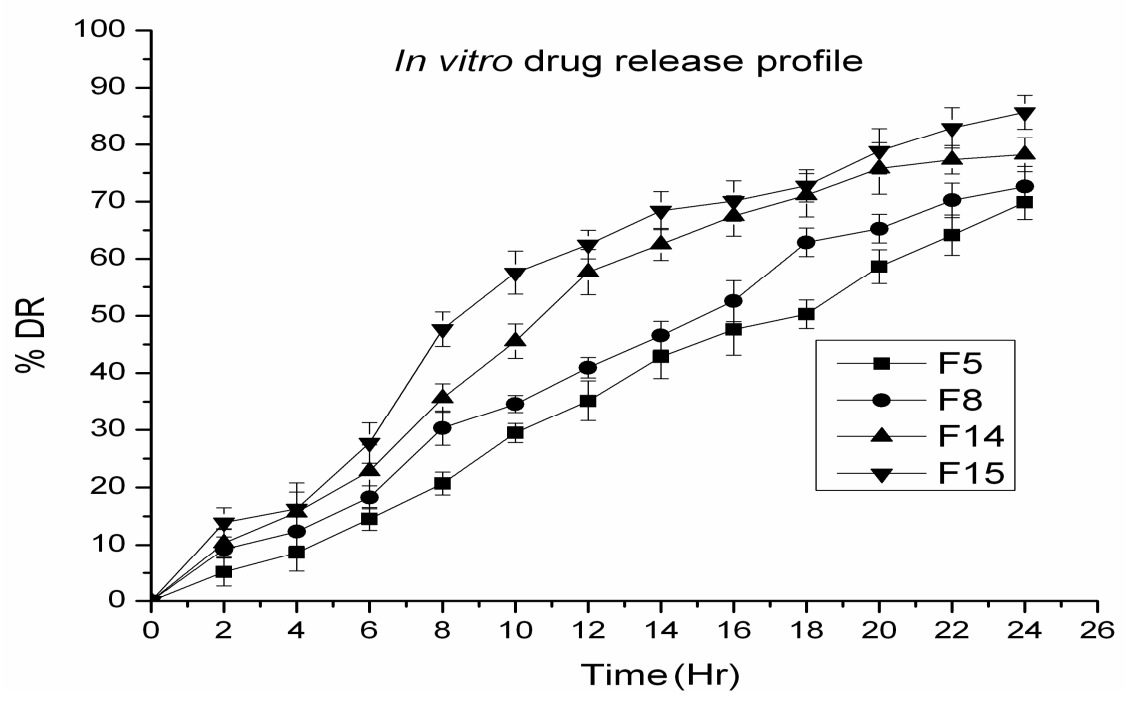

Figure 1: In vitro drug release profile of $M L X$ transdermal patches

The counter plots were constructed to elucidate the effect of dependent variables on the independent variable. The effect of $A$ and $B$ on drug release at DBT concentration of 20 is showed in Figure 2.

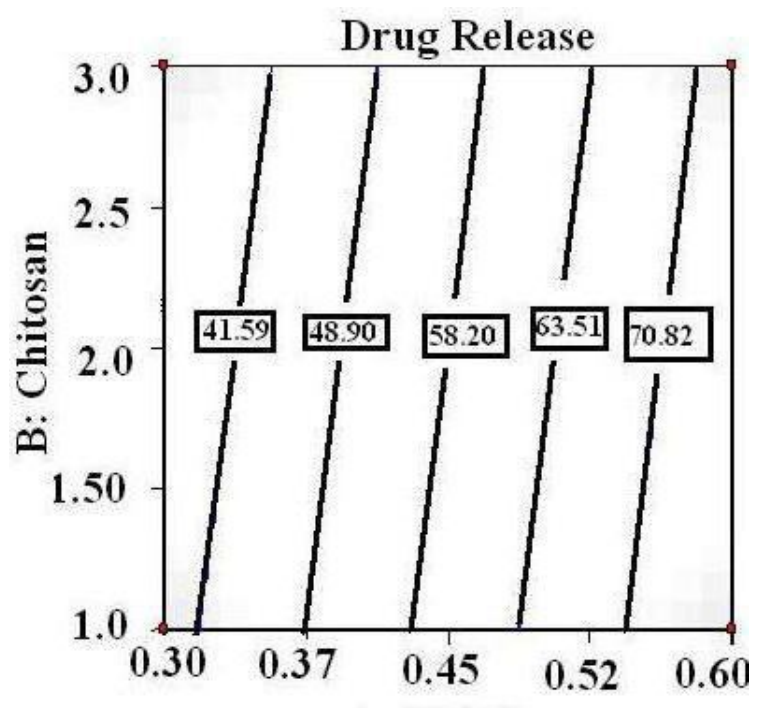

A: PAMA

Figure 2: Effect of PAMAM and chitosan on drug release of MLX from patches

As can be seen from counter plots, the drug release was increased as the concentration of PAMAM dendrimer in patches increases. When concentration of PAMAM was maintained at -1 level the drug release was increased from 30.4 $\%(\mathrm{~F} 6)$ to $45.1 \%$ (F17). At 0 level the further increase in drug release was found, in this case the drug release was increased from $50 \%$ (F9) to $62 \%$ (F10). $69 \%$ (F5) to $85 \%$ (F15) drug release was increased in case of +1 level. From the counter plot Figure 3, the effect of chitosan concentration on drug release could be predicted.

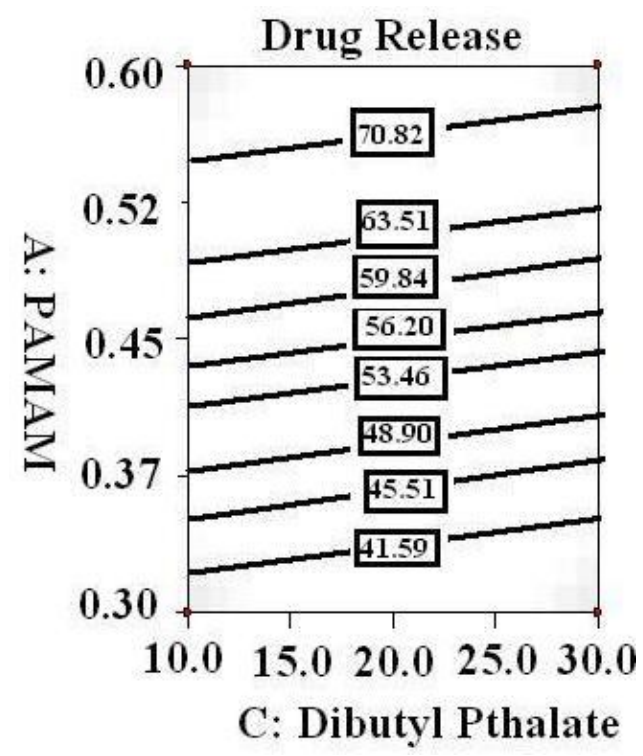

Figure 3: Effect of PAMAM and dibutyl pthalate on drug release of MLX from patches

When the concentrtion of chitosan was maintained at -1 level the drug release was found to be in the range of 45 (F17) to $85 \%$ (F15). At 0 level the decrease in drug release was observed and it was found to be the 33 (F1) to $78 \%$ (F14). Further decrease in drug release was observed when the chitosan concentration was maintain at +1 level and this case the drug release was 30.4 (F6) to $72 \%$ (F8). From Figure 3 it was clear that when concentration of DBT was maintained at - 1 level the drug release was found to be 42.23 (F11) to $78.24 \%(\mathrm{~F} 14)$ but when it was maintained at 0 level the drug release was 30.4 
$\%$ (F6) to $85.71 \%$ (F15). At +1 level the drug release was $33.25 \%$ (F1) to $69.9 \%$ (F5).

\section{Surface morphology and crystallinity}

FE-SEM photographs of the patches are shown in Figure $4 \mathrm{~A}$ and $\mathrm{B}$, and they display smooth and rough surface of the patches.

XRD study was performed to determine the crystalline and amorphous nature of the drug and polymer. It is clear from the Figure 5 that the crystalline peaks of the pure MLX have been disappeared in MLX loaded transdermal patches. Pure MLX was $78.30 \%$ crystalline and $21.7 \%$ amorphous in nature. But when MLX was entrapped in patch system along with dendrimer, chitosan and HPMC polymer the crystallinity of

MLX was decreased to $42.54 \%$. Therefore, in the final formulation, the MLX was $57.46 \%$ amorphous in nature.

\section{DISCUSSION}

The smoothness of the patches was attributed to the content of plasticizer in patches. The concentration of DBT imparted the smoothness and roughness to the patches. Some of the batches were found to be rough in nature but still the patches were pharmaceutically acceptable. The increase in thickness in patches was due to presence of backing layer and rate controlling membrane. The drug reservoir compartment in all batches was sandwiched between these two

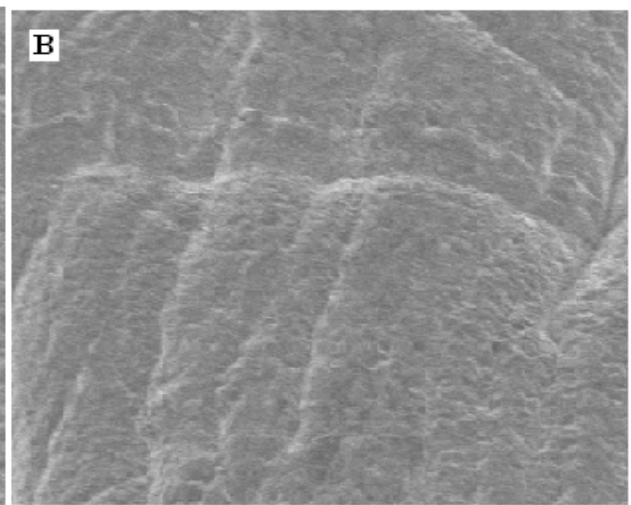

Figure 4: SEM of patches.

Note: $A$ = smooth surface without cracks, pores and wrinkles; $B=$ rough surface with wrinkles

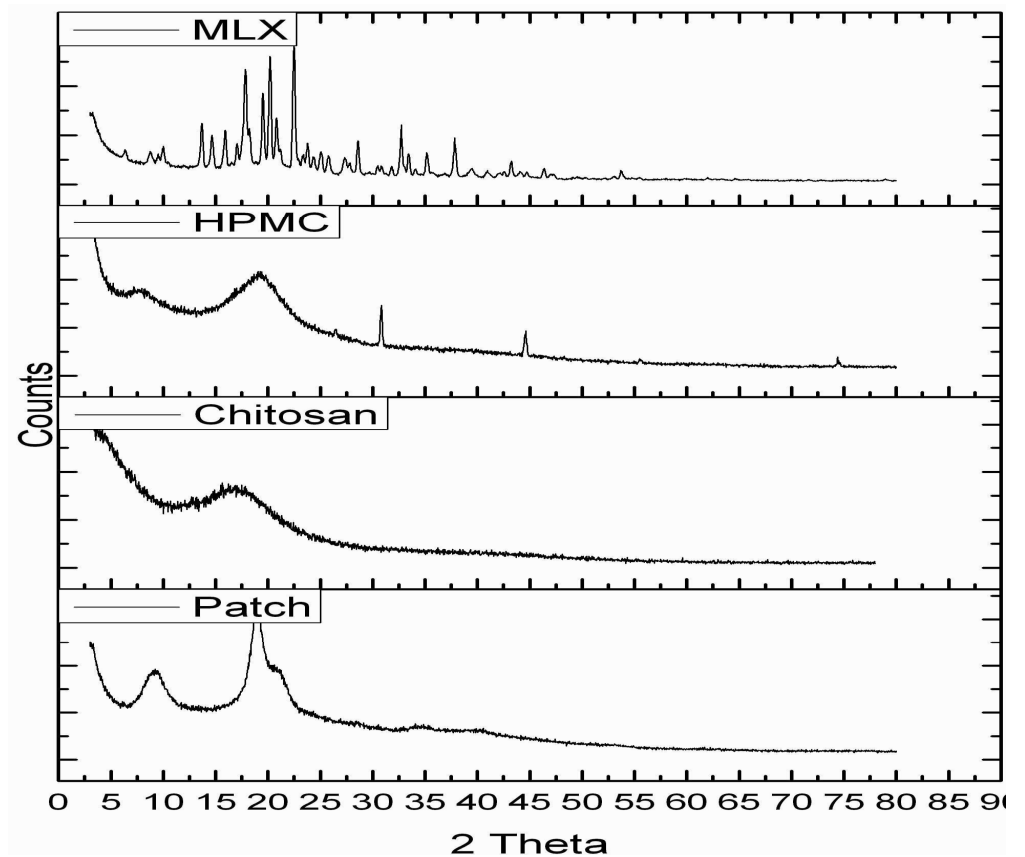

Figure 5: X-ray diffractograms showing various peaks of MLX, HPMC, chitosan and patch 
layers that lead to increase in thickness of the patches. Due to presence of hydrophilic HPMC polymer there were no any signs of crack on the patches. Also the presence of plasticizer DBT played imp role for the minimization of cracking of the patches. There was no any wide variation in weights of patches found. Nearly all patches were of the same weight. The possible reason for the good content uniformity in drug was may be due to minimum weight variation among the patches that lead to give uniformity in the drug content. These results obvious indicated that the solvent casting-evaporation technique employed to formulate was capable for producing patches with minimum weight variability with uniform drug content. The folding endurance test was performed to determine the ability of the patch to evaluate the flexibility and to endure rupture. This test indicates that the formulated patches would maintain integrity and would not break after application on the skin. The results showed higher folding endurance capacity with increasing concentration of the hydrophilic polymer blend system. It is clear that all the patches had low moisture content, which helped the formulations to remain stable and reduce cracking, wrinkling and brittleness during long term storage. Actually, a small amount of moisture content is always desirable to maintain the stability of the patches and protection of patches from the microbial contamination. From this study it was concluded that the increase in the concentration of hydrophilic chitosan/HPMC polymer was directly proportional to the increase in moisture uptake of the patches.

As the concentration of dendrimer increased the drug release from the patches also increased linearly. PAMAM dendrimers possesses empty internal cavities as well as amine functional groups on the outer shell. Empty internal cavities are able to encapsulate hydrophobic guest molecules in the macromolecule interior [14]. MLX could have been encapsulated by the empty internal cavities of PAMAM dendrimer. With amine terminated dendrimers, the proposed mechanism for solubility enhancement of MLX was electrostatic interactions between the terminal amine groups of dendrimers and $-\mathrm{OH}$ group of MLX and molecular encapsulation. So it is now clear that the linear increase in drug release was due to solubility enhancement effect of dendrimer on MLX. Extremely low water solubility of MLX could have been significantly improved by the PAMAM dendrimer that facilitated the drug release from the drug reservoir compartment of hydrophilic HPMC polymer. Though the effect of chitosan on drug release was not statistically significant, still we could predict that as the concentration of chitosan increased from the -1 level to +1 level, decrease in drug release was observed. Chitosan is hydrophillic polymer it forms gel when comes in contact with the water. Due to the gelling property of the chitosan in presence of aqueous medium, it forms the rate controlling layer for the patch system. So gelling of chitosan has retarded the release of MLX from the patch system. Also chitosan is pseudo plastic material which is excellent viscosity enhancing agent and viscosity increases with increase in chitosan concentration.

The combined effect of gelling property and viscosity enhancing effect of chitosan has controlled the release of MLX from the patch system. In case of plasticizer DBT there was no any linear relationship between concentration of DBT and drug release from the patches. The effect of DBT on drug release was statistically insignificant $(p=0.3274)$. It is clear from the SEM images that the prepered patches were having smooth surface as well as rough surface. In figure the wrinkles were observed on rough surface patches this may be due to the overdrying of the patches that laid to formation of rough appearance on the patches. The patches didn't show any sign of cracks or pores on the surface which reveals the cohesive structure of the polymer matrix. XRD results indicate that dendrimer probably changed $M L X$ to an amorphous form.

\section{CONCLUSION}

Dendrimers were found to be potential tool for the enhancement of the drug release from the patches. Dendrimer increased drug release of MLX by increasing the solubility and permeation through the membrane. Thus, dendrimer patches are a potential drug delivery system for the transdermal management of rheumatoid arthritis and possibly other related diseases.

\section{REFERENCES}

1. Amir G, Mehrzad H, Maryam S, Alireza K, Susan K, Mohammad RJN. Osteoprotegerin (OPG) and Matrix Gla protein (MGP) in rheumatoid arthritis patients: Relation to disease activity. The Egyptian Rheuma 2014; 36: 111-116.

2. Afrah S, Rami E, Afaf E, Ahmad S. Protein tyrosine phosphatase non-receptor type 22 (PTPN22) +1858 $C>T$ gene polymorphism in Egyptian cases with rheumatoid arthritis. Cell Immunol 2014; 290:62-65.

3. Joshi VR, Poojary VB. Cost-effective management of rheumatoid arthritis in India. Ind J Rheum 2013; 182187. 
4. Mazzenga GC, Berner B. The transdermal delivery of zwitterionic drugs I: the solubility of zwitterion salts. J. Control. Release 1991; 16:77-88.

5. Venkata $V$, Venuganti $K$, Omathanu $P$. Effect of poly(amidoamine) (PAMAM) dendrimer on skin permeation of 5-fluorouracil. Int J Pharm 2008; 361: 230-238.

6. Jain NK, Gupta U, Agashe HB, Asthana A. Dendrimers: Novel polymeric nanoarchitectures for solubility enhancement. Biomacromolecules 2006; 7(3)

7. Chauhan $A S$, Sridevi $S$, Chalasani KB, Jain $A K$ Jain SK, Jain NK, Diwana PV. Dendrimer-mediated transdermal delivery: enhanced bioavailability of Indomethacin. J. Control. Release 2003; 90(3): 335343.

8. Wang Z, Itoh Y, Hosaka Y, Kobayashi I, Nakano Y, Maeda I, Umeda F, Yamakawa J, Kawase M, Yag K. Novel transdermal drug delivery system with polyhydroxyalkanoate and starburst polyamidoamine dendrimer. J Biosci Bioeng 2003; 95(5): 541-543

9. Demiana I, Nesseem SF, Eid SS. Development of novel transdermal self-adhesive films for tenoxicam, an anti-inflammatory. Drug Life Sci 2011; 89: 430-438.
10. Mundada AS, Avari JG. Damarbatu as a novel matrix former for the transdermal drug delivery: in vitro evaluation. Drug Dev Ind Pharm 2009; 35(9): 11471154.

11. Samanta MK, Dube $R$, Suresh B. Transdermal drug delivery system of Haloperidol to overcome selfinduced extrapyramidal syndrome. Drug Dev Ind Pharm 2003; 29(4): 405-415.

12. Amnuaikit C, Ikeuchi I, Ogawara K, Higaki K, Kimura $T$. Skin permeation of propranolol from polymeric film containing terpene enhancers for transdermal use. Int J Pharm 2005; 289(1-2): 167-78

13. $O G D$ dissolution data available from: http://www.accessdata.fda.gov/scripts/CDER/dissoluti on/dsp_SearchResults_Dissolutions.cfm

14. Xin $H$, Zhenghong $W$, Wei $G$, Qin $C$, Bin $Y$. Polyamidoamine dendrimers as potential drug carriers for enhanced aqueous solubility and oral bioavailability of silybin Polyamidoamine dendrimers as drug carriers. Drug Dev Ind Pharm 2011; 37(4): 419-427. 